\title{
The midpoint of cortical thinning between late childhood and early adulthood differs across individuals and regions: Evidence from longitudinal modelling in a 12-wave sample
}

\author{
Fuhrmann, D. $*^{1}$, Madsen, K. S. ${ }^{2,3}$, Johansen, L. B. ${ }^{2}$, Baaré, W. F. C. ${ }^{+2}$ \& Kievit, R. A. ${ }^{+4}$
}

$*_{1}+$ Authors contributed equally

1 Department of Psychology, Institute of Psychiatry, Psychology, and Neuroscience, King's College London, London, UK

2 Danish Research Centre for Magnetic Resonance, Centre for Functional and Diagnostic Imaging and Research, Copenhagen University Hospital - Amager and Hvidovre, Kettegaard Allé 30, DK-2650, Hvidovre, Denmark

3 Radiography, Department of Technology, University College Copenhagen, Sigurdsgade 26, DK-2200, Copenhagen N., Denmark

4 Cognitive Neuroscience Department, Donders Institute for Brain, Cognition, and Behavior, Radboud University Medical Center, Nijmegen, The Netherlands 


\begin{abstract}
Charting human brain maturation between childhood and adulthood is a fundamental prerequisite for understanding the rapid psychological changes during adolescence. Two barriers have precluded the quantification of maturational trajectories: demands on data and demands on estimation. Using high-temporal resolution neuroimaging data of up to 12waves in the HUBU cohort ( $N=90$, aged 7-21 years) we investigate changes in apparent cortical thickness across childhood and adolescence. Fitting a four-parameter logistic nonlinear random effects mixed model, we quantified the characteristic, s-shaped, trajectory of cortical thinning in adolescence. This approach yields biologically meaningful parameters, including the midpoint of cortical thinning (MCT), which corresponds to the age at which the cortex shows most rapid thinning - in our sample occurring, on average, at 15 years of age. These results show that, given suitable data and models, cortical maturation can be quantified with precision for each individual and brain region.
\end{abstract}




\section{Introduction}

The human cortex undergoes protracted microscopic and macroscopic structural changes between childhood and adulthood ${ }^{1}$. Individual differences in cortical structure have been associated with a range of phenotypic differences, including physical and mental health ${ }^{2-5}$, neurodevelopmental disorders as well as cognitive performance in childhood and adolescence $^{2,6}$. Although other measures of brain structural development such as brain volume or white matter connectivity provide complementary insights into brain maturation, cortical thinning is one of the most widely used proxies of brain maturation ${ }^{7-9}$. Studies generally observe developmental decreases in cortical thickness from childhood to early adulthood $^{8}$, with an emerging consensus on a characteristic s-shaped, non-linear trajectory ${ }^{9,10}$ at the population level (Figure 1). This process of cortical thinning is thought to reflect a range of underlying biological processes ${ }^{11}$, including increasing myelination of the deeper cortical layers ${ }^{12}$ and decreasing synaptic density ${ }^{13}$.

Hypotheses concerning individual differences in the process of cortical thinning and their link to psychological development feature prominently in neurodevelopmental theories: For instance, it has been hypothesized that a life history of adversity may lead to accelerated $^{14,15}$ or delayed ${ }^{16,17}$ cortical maturation. The developmental mismatch theory suggests that a mismatch in maturity between subcortical and cortical brain regions (with frontal regions commonly thought to thin last) may help explain the prevalence of risk-taking behaviour during adolescence when this maturation disparity is thought to be maximal ${ }^{18,19}$. At the group comparison level, hypotheses posit that girls demonstrate earlier cortical maturation than boys ${ }^{20-22}$, with these differences hypothesized to underlie developmental differences in behavioural and psychopathological phenotypes. Similarly, Nunes et al. (2020) hypothesized that children with autism spectrum disorder are characterized by accelerated brain maturation ${ }^{23}$. Overall, hypothesized differences in cortical maturation are central to some of the most influential neurodevelopmental theories. However, the data and analytic methods currently used to capture maturation are no match for ambitions in understanding and applying the construct of cortical maturation. 


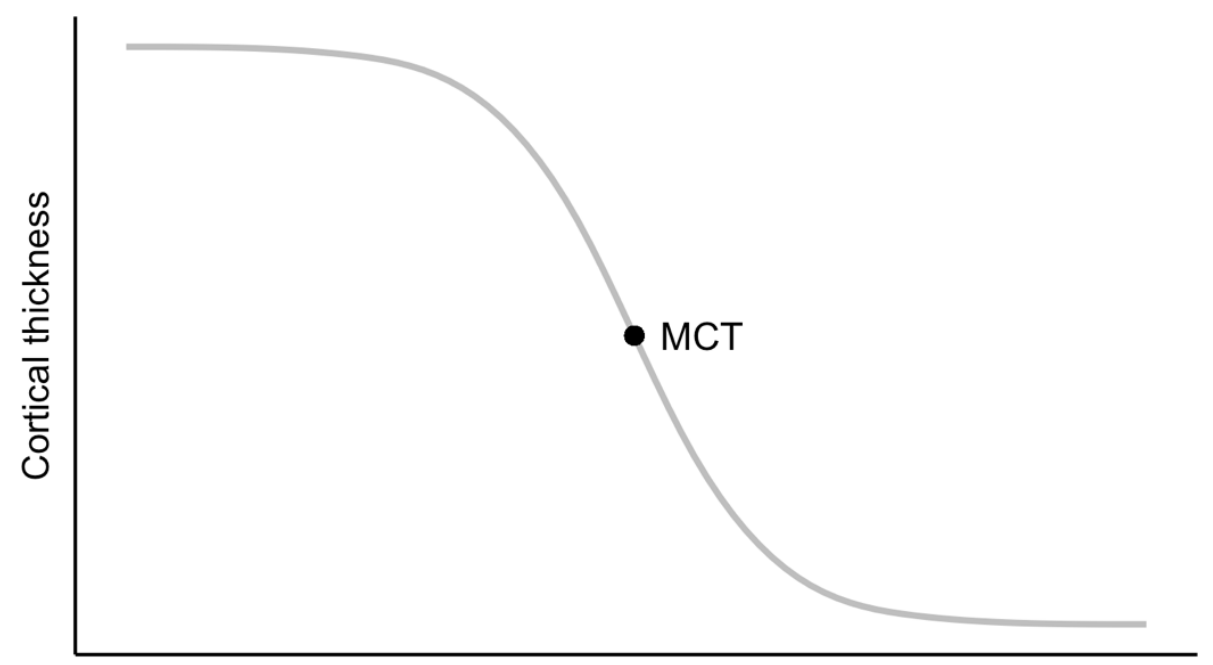

Age (late childhood to early adulthood)

Figure 1. Schematic illustration of cortical thinning during adolescence, showing the Midpoint of Cortical Thinning (MCT), i.e., the age with the most rapid cortical thinning, between late childhood and early adulthood.

Most neuroimaging studies to-date rely on cross-sectional data, as longitudinal data is costly and time-consuming to procure. Cross-sectional data precludes the investigation of developmental changes and necessitates researchers use an indirect proxy such as apparent cortical thickness to reflect the relative developmental stage of individuals. The limitations of this practice are considerable, as any cross-sectional measure necessarily conflates distinct sources of cortical thickness differences (baseline thickness, the onset of maturation, as well as speed and total amount of thinning). Only under extremely restrictive assumptions (e.g., identical brain thickness in early childhood and late adolescence, identical rates of thinning) can a cross-sectional measure be used as a proxy for development. Given that these assumptions are known to be empirically untrue ${ }^{1}$, our knowledge of cortical maturation is likely to be extremely limited.

Where longitudinal data does exist, it often consists of a small (2 or 3 ) number of waves $^{9,24}$. Most longitudinal studies investigating individual differences to date have used linear modelling approaches using the percentage of, or absolute change, in cortical thickness between two ages as an indicator of maturation of a given brain region, with larger changes commonly equated to more protracted maturation ${ }^{25,26}$. However, these estimates are typically confounded by the initial thickness of a region - thicker regions can thin more than thinner regions. Even when such confounds are controlled for, the absolute change in 
thickness remains dependent on the precise age range studied. Finally, the number of waves limits our ability to capture the trajectory of cortical thinning, which is known to be nonlinear ${ }^{27}$. This is particularly true at the individual level.

To address the challenge of quantifying cortical maturation at the individual level, we here leverage a unique dataset (with up to 12 longitudinal measurements between late childhood and early adulthood) and a quantitative framework currently underutilized in cognitive neuroscience (non-linear random effects modelling) to demonstrate that cortical maturity can be defined, and estimated, at the individual level, offering a new window of insight into cortical development across adolescence. Nonlinear mixed models are a powerful tool that can capture developmental processes at the individual level ${ }^{28}$ and yield readily interpretable parameters. One of these parameters allows us to provide a novel quantitative definition of cortical maturation during adolescence at the individual level: The midpoint of cortical thinning (MCT, see Figure 1). The MCT is the point in adolescent development where the rate of cortical thinning is at its peak for an individual and thus slows down afterwards. We show that the MCT can be used to study the extent to which cortical maturation differs between individuals, genders, and brain regions.

\section{Results}

Cortical thickness showed nonlinear changes between childhood and early adulthood across all cortical regions (Supplementary Figure 1). The characteristic sigmoid, or s-shaped, curve, of cortical thinning, was apparent across most brain regions. We captured this shape using the four-parameter logistic function:

$$
y=A_{\text {Lower }}+\frac{A_{\text {Upper }}-A_{\text {Lower }}}{1+\left(\frac{x}{\text { Inflection }}\right)^{- \text {Hill }}}
$$

The function yields biologically meaningful parameters: The upper asymptote ( $A_{\text {upper, }}$ maximal apparent thickness in $\mathrm{mm}$ ), lower asymptote $\left(A_{\text {Lower, }}\right.$ minimal apparent thickness in $\mathrm{mm}$ ), and Hill, the slope of change). We were particularly interested in the inflection point, which corresponds to the MCT and was here used as an index of cortical maturation (see Figure 1). The MCT can be compared across individuals, genders, and brain regions. To this end, we first modelled cortical thickness averaged across the cortex, identified the average 
pattern of maturation, and assessed gender differences. Next, we modelled cortical thickness for different brain regions to investigate patterns of maturation across the cortex. Gender and in-scanner motion, as a proxy for image quality, was controlled for in all analyses.

\section{There are substantial individual differences in the MCT}

We found that the nonlinear model fit the data well (Supplementary Figure 2). Mean cortical thickness was estimated with very good precision for the asymptotes, MCT, and the hill, as indicated by low coefficients of variation $\left(C V_{i}\right.$ Supplementary Table 1$)$. The MCT was uncorrelated with the upper asymptote, showing that it is independent of the initial thickness of a region $(r=0.08, t(88)=0.78, p=.438)$. It was also independent of the mean cortical thickness in early adulthood $\left(A_{\text {Lower, }} r=0.16, t(88)=1.47, p=.145\right)$. This independence means that cross-sectional measurements of cortical thickness will not function well as an approximation of cortical maturity. The rate of change (Hill) was associated moderately with the MCT $(r=-0.26, t(88)=-2.54, p=.013)$. This means that a later MCT was associated with a flatter curve overall.

Because the sigmoid is asymptotic, there is no age at which the brain is mature (Figure $2 A)$. Instead, the brain develops throughout the age range investigated here ( $7-21$ years). Parameters indicated that mean thickness showed stable and high levels in late childhood, with an upper asymptote of $2.95 \mathrm{~mm}(S E=0.01 \mathrm{~mm})$ and decreasing thereafter to a lower asymptote of $2.59 \mathrm{~mm}(S E=0.04 \mathrm{~mm})$. The overall amount of apparent thinning ( $A_{u p p e r}-$ $A_{\text {Lower }}$ ) between ages 7-21 years was estimated to be $0.36 \mathrm{~mm}$ (Supplementary Table 1). Our central parameter of interest, the $\mathrm{MCT}$, was estimated to be 14.72 years ( $S E=0.30$ years). We observed a substantial range of MCTs across individuals, with a minimum and maximum of 12.41 and 19.21 years (Figure $2 \mathrm{~A} \& 2 \mathrm{~B}$ ) and a variance of 1.75 years ( $S E=0.47$ years). Together, our analysis demonstrates that we can estimate a novel, quantitative definition of cortical maturity which is independent of overall thickness and shows substantial differences between people. 

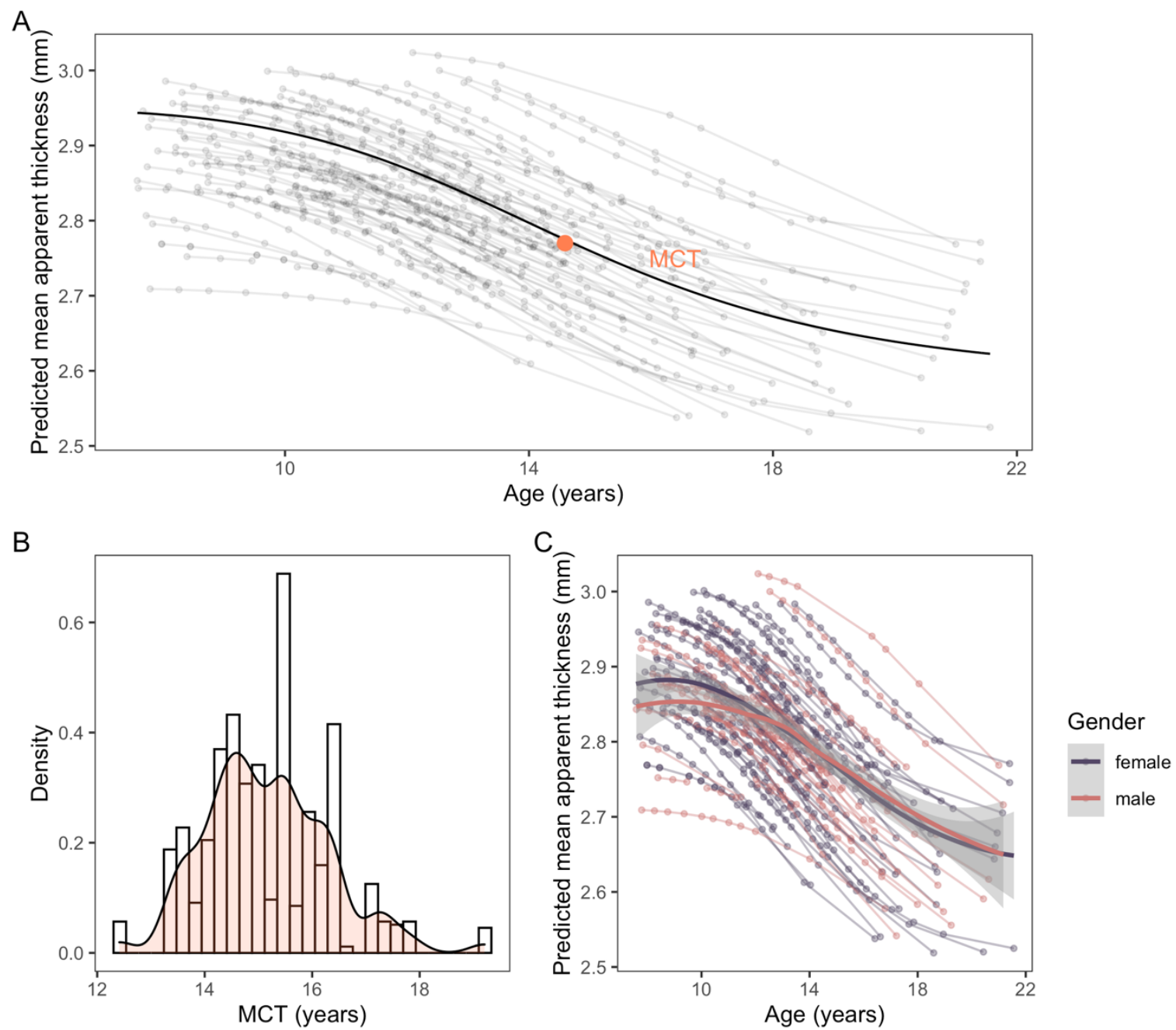

Figure 2. Panel $A$ : Predicted apparent mean cortical thickness as a function of age. Estimated individual trajectories and the average trajectory is shown, as well as the average MCT. Panel B: Density plot of MCTs showing individual differences in the sample. Panel C: Gender differences in the sample. MCT = Midpoint of Cortical Thinning.

\section{Significant, but noisy, gender differences in trajectories}

There were significant gender differences: Females started with thicker cortices than males $(b=-0.06, p<.001)$ and showed earlier MCT $(b=1.32, p<.001$, Figure $2 C)$. However, the CVs for these estimates were $31.0 \%$ and $31.4 \%$ respectively, indicating that these parameters were estimated with low precision. The low precision was likely due to the small number of males in the sample $\left(N_{\text {Males }}=37\right)$. 
bioRxiv preprint doi: https://doi.org/10.1101/2022.02.10.479868; this version posted February 10, 2022. The copyright holder for this preprint (which was not certified by peer review) is the author/funder, who has granted bioRxiv a license to display the preprint in perpetuity. It is made available under aCC-BY 4.0 International license.

\section{MCTs differ across the cortex}

To examine the specificity in maturational timing across regions, we estimated the MCT for each of the 34 cortical regions of the Desikan-Killiany atlas independently (Figure 3), again using non-linear mixed models and the four-parameter logistic function. The four parameters were estimated with good precision $(C V<20 \%)$ for all regions ${ }^{29}$, except for the entorhinal, lingual, parahippocampal, pericalcarine, temporal pole, and transverse temporal region, which were all excluded from subsequent analyses (see Supplementary Table 2 and 3 for all estimates). Mean MCTs ranged from 13.91 years for the frontal pole to 17.48 years for the postcentral region (Figure $3 \mathrm{~A}$ ).

Next, we implemented a formal quantitative test to examine whether regions differed in their MCTs. To do so, we compared a confirmatory factor model with intercepts for MCTs across regions constrained to equality (reflecting the hypothesis that regions mature at the same approximate age) to a model where they are estimated freely, reflecting the hypothesis that regions mature at distinct ages). Despite the considerable added complexity, we found that allowing MCTs to differ between regions, substantially improved model fit $\left(\Delta \mathrm{X}^{2}(28)=857.97, p<.001\right)$ indicating pronounced differences in maturational timing between regions independent of their overall thickness.

In addition to MCT-differences across regions globally, we also examined whether MCTs are linked between some or all regions: In other words, if a person is mature in one region, are they then also more mature in all other regions, or are there clusters of brain regions that covary in their relative maturity? To examine this question, we first examined the fit of a one-factor confirmatory model, testing the hypothesis that a single factor could capture MCTs across the brain. This model fit poorly $\left(X^{2}(350)=875.25, p<.001, C F I=0.839\right.$, SRMR $=0.053$, RMSEA $=0.129[0118,0.140])$. To determine whether a more complex model might fit the data, we used Exploratory Factor Analysis. Eigenvalues of a parallel analysis suggested that a single factor yields the best, albeit imperfect, solution $\left(X^{2}(350)=760.17, p<\right.$ .001). The single factor explained $66.9 \%$ of the variance in MCTs. Most regions loaded strongly onto the factor (Figure 3B, Supplementary Table 4). The rostral anterior cingulate and frontal pole showed the lowest loadings by a margin, with 0.235 and 0.315 respectively. Finally, we explored whether there is a regional ordering in the timing of maturation in line with hypotheses of a posterior-to-anterior gradient across the cortex ${ }^{7,30}$. We correlated 
inflection ages of each region with their posterior-anterior coordinate in MNI space. We found no evidence for a significant correlation between the spatial location and the inflection ages $(t(260)=-0.25, p=.809, r=-0.05$, Bayes Factor $=0.36)$.

Together, these analyses offer new insight into cortical maturation. We demonstrate, for the first time, that it is possible to estimate non-linear maturation independent of overall cortical thickness. Maturational trajectories differed between individuals and cortical regions. The ability to estimate these differences offers a new window into elucidating longstanding debates concerning the speed of maturation, its association with early adversity, and the implications for cognitive and mental health development. 
A

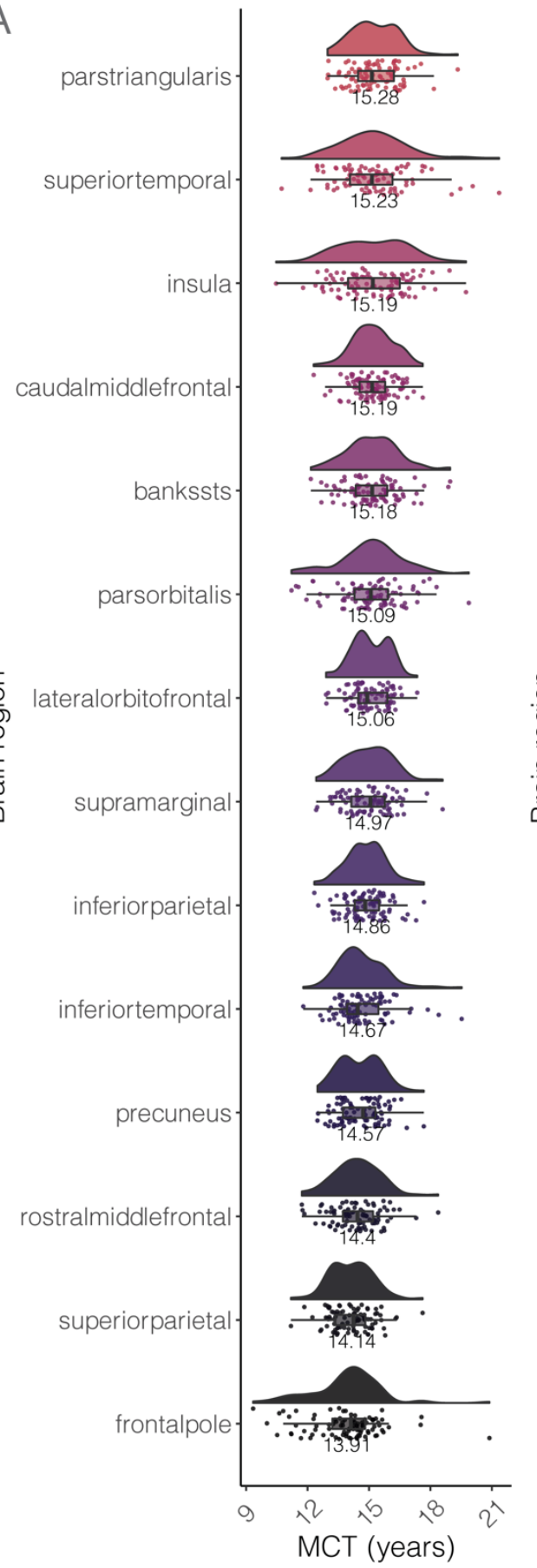

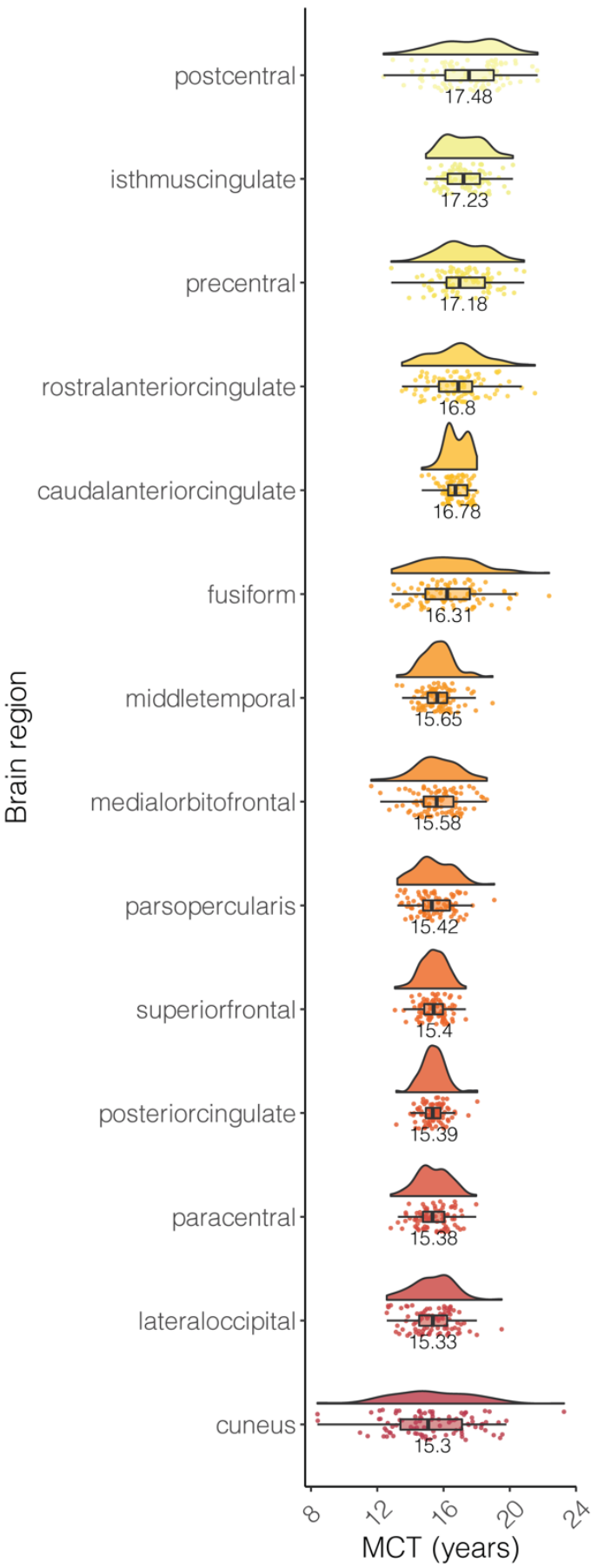

B

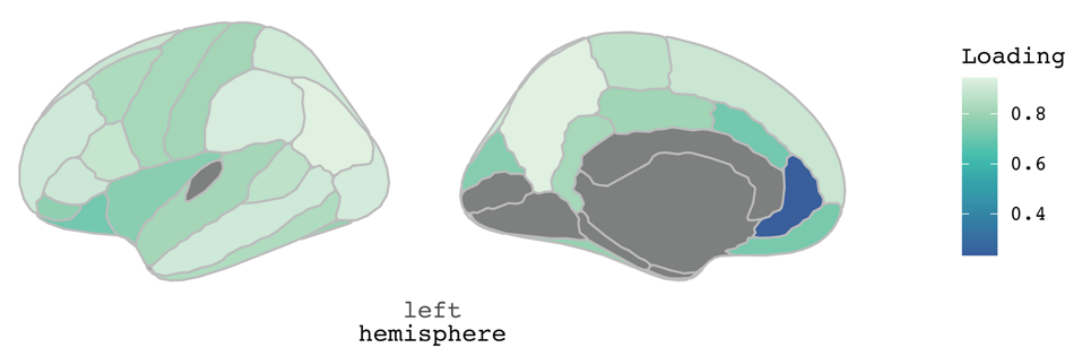

Figure 3. Panel A: Individual and regional differences in thinning. Panel $B$ : Maturational factor loadings plotted across the cortex, as identified by the exploratory factor analysis. 


\section{Discussion}

Using longitudinal data of up to 12-waves imaged between late childhood and early adulthood and flexible nonlinear mixed models, we here show that cortical maturity as indexed by the MCT can be separated from other cortical thickness parameters (i.e., asymptotes and slope - hill) and estimated precisely and reliably for each individual and most brain regions. We identified a characteristic, s-shaped, trajectory of cortical thinning: Cortical thickness was high and stable in childhood, followed by decreases in early adolescence, culminating around the age of 15 years, the average MCT. The reduction in cortical thickness then decelerates to level off in late adolescence. This finding is in line with previous studies showing s-shaped reductions in cortical thickness over adolescence ${ }^{9,10}$ and extend the same by providing estimates of upper limits in apparent cortical thickness (2.95 $\mathrm{mm})$, lower limits of apparent cortical thickness (2.54 mm), and, most importantly, an index of maturation: the MCT (average of 14.72 years). This highlights that cortical thinning is protracted across adolescence and shows rapid changes in adolescence. This period of rapid brain development raises questions about possible sensitive periods in adolescence ${ }^{31,32}$. Future research will be able to show whether periods of structural change confer heightened plasticity in adolescence.

Developmental patterns differed between cortical regions, with frontal regions such as the rostral middle frontal gyrus, showing the earliest MCTs, around 14 years, and cingulate regions, showing some of the latest MCTs, around 17 years. Early maturing regions were found in lateral frontal, and parietal areas, while late-maturing regions were found in temporal and dorsal central areas. Along with recent work identifying structural brain networks and hubs ${ }^{33^{-36}}$, this finding supports a complex systems account, in which the brain matures in a distributed pattern. Future studies could use complex systems approaches like network models to identify how maturation across regions produces changes in cognition and mental states across development.

We found evidence for pronounced individual differences, with MCTs differing by several years between individuals. This supports the notion that brain maturation is highly variable $^{16,17,37}$ and invites questions of potential predictors and outcomes. We here investigated gender as a potential predictor of individual differences. There was some evidence for gender differences, with females showing earlier maturation than males, by 
about 1 year. This supports initial evidence for earlier maturation in girls from older longitudinal studies ${ }^{38,39}$ and may be linked to earlier puberty and different socialization in girls ${ }^{40,41}$. Our estimates of gender differences were relatively noisy, however. Future studies could use nonlinear mixed models in larger cohorts, such as $A B C D^{42}$, to investigate the robustness of gender differences in maturation. Future studies could also investigate other candidate predictors such as environmental influences (e.g., adversity and education) to identify whether these accelerate or decelerate maturation - a yet unsolved conundrum in developmental science ${ }^{16,17,37}$. Investigations of potential outcomes of maturational differences, e.g., cognitive performance or psychiatric diagnosis, would be similarly fascinating, and could eventually include distal effects, such as cognitive and brain changes during ageing ${ }^{1,43}$.

It is worth noting that our analytical approach, using the four-parameter logistic function, depends on the nature of cortical thinning: Other measures of brain maturation (e.g., brain volume) likely show different developmental shapes and different maturational timelines, not all of which will be amenable to estimating the MCT. The simple linear decreases, reported for volume changes in some cortical regions, would not allow for a quantitative 'midpoint.' However, nonlinear mixed models are extremely versatile and can be fit, in principle, to any functional relationship, even a linear one. In the future, these tools can be used to model white matter trajectories and other morphometric measures of cortical and subcortical development to better understand similarities and differences across brain tissues. This will yield a precise understanding of how changes in grey and white matter work in concert to produce functional changes in the brain and behaviour.

In conclusion, this study shows that apparent cortical thinning in adolescence is sshaped, with the most rapid changes occurring in mid-adolescence, at around 15 years of age, on average. Further, we show that individuals vary substantially, with up to several years, in the age at which the cortex undergoes most rapid changes. On a practical level, this work shows that high-resolution temporal data, combined with nonlinear modelling approaches, can be used to quantify brain maturation with unprecedented precision. This will allow the field to provide rigorous tests of prominent theoretical models of adolescent development, such as the structural mismatch hypothesis ${ }^{18,19}$ or accelerated maturation hypotheses ${ }^{15}$. 


\section{Materials and Methods}

\section{Cohort}

The present longitudinal study included data from 90 typically developing children and adolescents (53 females, 37 males), who were enrolled in the longitudinal HUBU ("Hjernens Udvikling hos Børn og Unge", Brain Maturation in Children and Adolescents) study. The HUBU study was initiated in 2007, where 95 participants ( 55 females, 40 males) aged 7 - 13 years and their families were recruited from three elementary schools in the Copenhagen, DK, suburban $a^{2} a^{44}$. All children whose families volunteered were included, except for children with a known history of neurological or psychiatric disorders or significant brain injury. Children assented to the study procedures and informed written consent was obtained from parents. Informed written consent was also obtained from the participants themselves when they turned 18 years of age. The study was approved by the Ethical Committees of the Capital Region of Denmark (H-KF-01-131/03 and H-3-2013-037) and performed in accordance with the Declaration of Helsinki. Participants were scanned up to 12 times with scanning intervals of 6 months for the first 10 visits, one year between visits 10 and 11 , and three years between visits 11 and 12 (Supplementary Figure 3).

Here, we included data from the first 12 assessments of the HUBU study. All baseline MRI scans were evaluated by an experienced neuroradiologist, and all raw images were visually inspected to ensure sufficient quality. Five participants were excluded from the present study, due to receiving a psychiatric diagnosis after study initiation $(N=2)$, incidental clinical finding on the MRI scan $(N=1)$, or no MRI scans or FreeSurfer outcomes of sufficient quality $(N=2)$. Our final sample analysed here consisted of 90 participants ( 53 females, 37 males) aged 7.6 - 21.6 years. For this sample, we excluded 73 MRI sessions if one of the following criteria was met: participants did not finish the MRI session (2 participants, 2 scans), participants were not scanned due to metallic dental braces (14 participants, 31 scans), had poor MR-image quality (22 participants, 31 scans), or had acquired a brain injury after baseline (1 participant, 9 scans). A total of 745 valid MRI scans (scans per participant: range $=1-12$, mean $=8.3$, median $=10$, interquartile range $(I Q R)=8-12$ ) were included in the statistical analyses. A summary of the longitudinal scans and participants' age at each scan is shown in Supplementary Figure 3. Data from the HUBU cohort has previously been 
used in cross-sectional ${ }^{45-49}$ and longitudinal ${ }^{44,50}$ studies examining brain-behavioural relationships.

\section{MRI protocol}

Participants underwent structural MRI on a $3^{T}$ Siemens Magnetom Trio MR scanner (Siemens, Erlangen, Germany) using an eight-channel head coil (Invivo, FL, USA). Two T1weighted images were acquired using a 3D MPRAGE sequence $(T R=1550 \mathrm{~ms}$, TE $=3.04 \mathrm{~ms}$, matrix $=256 \times 256,192$ sagittal slices, $1 \times 1 \times 1 \mathrm{~mm}^{3}$ voxels, acquisition time $\left.=6: 38\right)$. A T2weighted image was acquired using a $3 D$ turbo spin echo sequence $(T R=3000 \mathrm{~ms}, T E=$ $354 \mathrm{~ms}$, FOV $=282 \times 216$, matrix $=256 \times 196,192$ sagittal slices, $1 \times 1 \times 1 \mathrm{~mm}^{3}$ voxels, acquisition time $=8: 29$ ).

\section{FreeSurfer pre-processing and extraction of cortical thickness}

All T1-weighted and T2-weighted images were processed using tools available in the FreeSurfer (version 6.0) software suite ${ }^{51-53}$. Cortical surface reconstruction was implemented using the following procedures: skull stripping, non-uniformity correction, white matter segmentation, creation of initial mesh, correction of topological defects, and creation of optimal white and pial surfaces ${ }^{51-53}$. Images were then processed with the longitudinal stream $^{54}$ in FreeSurfer, to estimate changes in cortical thickness across time. Apparent cortical thickness was calculated as a measure of the shortest distance between the white and pial surfaces. Cortical grey matter parcellations were based on surface-based nonlinear registration to the Desikan-Killiany atlas based on gyral and sulcal patterns and Bayesian classification rules ${ }^{53}$, yielding estimates for 34 ROls in each hemisphere. Cortical thickness estimates were averaged across the hemispheres.

To quality check the FreeSurfer outputs, we used an approach like that used in Enigma (http://enigma.ini.usc.edu/protocols/imaging-protocols/), i.e., statistical detection of outliers for the average thickness and surface area of each cortical parcel, which were then visually inspected. The statistical detection of outliers was performed on the age and sexadjusted residuals, to account for age and sex differences in the brain measures. 
Furthermore, to conform to the Enigma protocol, we did not perform manual editing on the FreeSurfer 6 outputs and discarded data of questionable quality.

Finally, the Euler number, which indicates the topological complexity of the reconstructed cortical surface, was extracted from FreeSurfer for each scan and used as a quantitative measure of image data quality in our statistical analyses, to account for potential systematical bias in data quality across age. The Euler number has been correlated with visual image quality inspection scores as well as with cortical thickness in a regionally heterogeneous pattern across datasets ${ }^{55}$. The Euler number was extracted for each hemisphere and summed to produce one value per scan.

\section{Statistical analyses}

We here modelled cortical thinning between childhood and adulthood using nonlinear mixed models implemented via the saemix ${ }^{56}$ package (version 2.4 ) in R (version 4.1.0) and RStudio (version 1.4.1717). To capture the characteristic s-shape of cortical thinning, we fit the fourparameter logistic function ${ }^{57}$, as defined in the Results section. See https://github.com/df1234/HUBU_NLMMs for our analysis code. Cortical thickness was modelled as the dependent variable and age as the independent variable. We fit a model to mean cortical thickness, as well as for one for each of the 34 ROIs of the Desikan-Killiany atlas $^{53}$. We included gender and Euler numbers as covariates in all models, to account for potential differences thereof in developmental trajectories ${ }^{38,39}$. In NLMMs, covariate parameters can be included to control for, e.g., the effect of motion, on any of the main parameters of the model (here the asymptotes, the inflection, and the hill). To avoid overfitting, we determined the covariate model via forward model selection. We allowed the upper asymptote and MCTs to differ between genders and included the motion parameter as a covariate for the lower asymptote. The remaining parameters were held constant for gender and motion. Estimates were obtained for the four parameters of the logistic function, as well as the two covariates. Precision was assessed by inspecting the coefficient of variation (CV) for each parameter, as provided by saemix. CVs are standardized measures of dispersion and are calculated as the ratio of the standard deviation to the mean. CVs $<20 \%$ are generally considered acceptable ${ }^{29}$. 
In a second step, we assessed differences in MCT estimates across different brain regions. We assessed whether MCTs across brain regions could be constrained to equality using Confirmatory Factor Analysis (CFA), as implemented in lavaan ${ }^{58}$ for R (version 0.6-9). We used full information maximum likelihood with robust standard errors to account for missingness and nonnormality. We estimated a one-factor CFA model in which factor loadings were freely estimated for each brain region. We then compared this model to one where the factor loadings were still freely estimated, but the intercepts were constrained to equality using the likelihood ratio test. A significant likelihood ratio test here indicates a loss in model fit for a constrained model, providing evidence that brain regions differ in their MCTs.

Third, we used Exploratory Factor Analysis to assess the dimensionality of MCTs across regions and to identify maturational factors capturing developmental trends across regions. This was implemented through parallel analysis via the $\mathrm{psych}^{59}$ package for $\mathrm{R}$ (version 2.1.6), using an oblique oblimin transformation.

Finally, we tested the popular hypothesis of a posterior-anterior gradient of development ${ }^{60}$ by correlating MCTs for each region with their y-coordinates in MNI space. Coordinates were obtained from the brainGraph ${ }^{61}$ package in $\mathrm{R}$ (version 3.0.0).

\section{Acknowledgements}

The authors thank the children and young people and their parents for their participation in the HUBU study. The HUBU project was supported by the Danish Council of Independent Research | Medical Sciences (grant numbers 09-060166 and 0602-02099B), the Lundbeck Foundation (grant number R32-A3161), and the Lundbeck Foundation Center of Excellence grant to The Center for Integrated Molecular Brain Imaging. DF was supported by the UK Economic and Social Research Council (ES/T015861/1). RAK was supported by a Hypatia Fellowship at the RadboudUMC.

\section{Conflict of Interest Statement}

The authors report no conflict of interest. 


\section{References}

1. Bethlehem, R. A. I. et al. Brain charts for the human lifespan. bioRxiv 2021.06.08.447489 (2021) doi:10.1101/2021.06.08.447489.

2. Fuhrmann, D., Simpson-Kent, I. L., Bathelt, J., The CALM Team \& Kievit, R. A. A hierarchical watershed model of fluid intelligence in childhood and adolescence. Cerebral Cortex (2019) doi:10.1093/cercor/bhzog1.

3. Humphreys, K. L. et al. Stressful Life Events, ADHD Symptoms, and Brain Structure in Early Adolescence. Journal of Abnormal Child Psychology 47, 421-432 (2019).

4. Mofrad, S. A., Lundervold, A. J., Vik, A. \& Lundervold, A. S. Cognitive and MRI trajectories for prediction of Alzheimer's disease. Scientific Reports 11, 2122 (2021).

5. Bos, M. G. N., Peters, S., van de Kamp, F. C., Crone, E. A. \& Tamnes, C. K. Emerging depression in adolescence coincides with accelerated frontal cortical thinning. Journal of Child Psychology and Psychiatry 59, 994-1002 (2018).

6. Shaw, P. et al. Intellectual ability and cortical development in children and adolescents. Nature 440, 676-679 (2006).

7. Squeglia, L. M., Jacobus, J., Sorg, S. F., Jernigan, T. L. \& Tapert, S. F. Early adolescent cortical thinning is related to better neuropsychological performance. Journal of the International Neuropsychological Society : JINS 19, 962-70 (2013).

8. Walhovd, K. B., Fjell, A. M., Giedd, J., Dale, A. M. \& Brown, T. T. Through Thick and Thin: a Need to Reconcile Contradictory Results on Trajectories in Human Cortical Development. Cerebral Cortex 27, (2017).

9. Tamnes, C. K. et al. Development of the cerebral cortex across adolescence: A multisample study of interrelated longitudinal changes in cortical volume, surface area and thickness. Journal of Neuroscience (2017) doi:10.1523/JNEUROSCI.3302-16.2017. 
10. Ducharme, S. et al. Trajectories of cortical thickness maturation in normal brain development--The importance of quality control procedures. Neuroimage 125, 267-279 (2016).

11. Jernigan, T. L., Baare, W. F., Stiles, J. \& Madsen, K. S. Postnatal brain development: structural imaging of dynamic neurodevelopmental processes. Progress in brain research 189, 77-92 (2011).

12. Natu, V. S. et al. Apparent thinning of human visual cortex during childhood is associated with myelination. Proc Natl Acad Sci USA 116, 20750 (2019).

13. Huttenlocher, P. R. Synaptic density in human frontal cortex - Developmental changes and effects of aging. Brain research 163, 195-205 (1979).

14. Keding, T. J. et al. Differential Patterns of Delayed Emotion Circuit Maturation in Abused Girls With and Without Internalizing Psychopathology. AJP 178, 1026-1036 (2021).

15. Belsky, J. Early-Life Adversity Accelerates Child and Adolescent Development. Curr Dir Psychol Sci 28, 241-246 (2019).

16. Tooley, U. A., Bassett, D. S. \& Mackey, A. P. Environmental influences on the pace of brain development. Nature Reviews Neuroscience 22, 372-384 (2021).

17. Colich, N. L., Rosen, M. L., Williams, E. S. \& McLaughlin, K. A. Biological aging in childhood and adolescence following experiences of threat and deprivation: A systematic review and meta-analysis. Psychol Bull 146, 721-764 (2020).

18. Steinberg, L. Risk taking in adolescence: what changes, and why? Ann. N. Y. Acad. Sci. $1021,51-58(2004)$.

19. Casey, B. J., Getz, S. \& Galván, A. The adolescent brain. Dev Rev 28, 62-77 (2008). 
20. Giedd, J. N. et al. Brain development during childhood and adolescence: A longitudinal MRI study. Nature neuroscience 2, 861-863 (1999).

21. Vijayakumar, N. et al. Thinning of the lateral prefrontal cortex during adolescence predicts emotion regulation in females. Soc Cogn Affect Neurosci 9, 1845-1854 (2014).

22. Peper, J. S., Burke, S. M. \& Wierenga, L. M. Chapter 3 - Sex differences and brain development during puberty and adolescence. in Handbook of Clinical Neurology (eds. Lanzenberger, R., Kranz, G. S. \& Savic, I.) vol. 175 25-54 (Elsevier, 2020).

23. Nunes, A. S. et al. Atypical age-related changes in cortical thickness in autism spectrum disorder. Scientific Reports 10, 11067 (2020).

24. Vijayakumar, N. et al. Brain development during adolescence: A mixed-longitudinal investigation of cortical thickness, surface area, and volume. Hum Brain Mapp 37, 2027$2038(2016)$.

25. Storsve, A. B. et al. Differential longitudinal changes in cortical thickness, surface area and volume across the adult life span: regions of accelerating and decelerating change. J Neurosci 34, 8488-8498 (2014).

26. Schnack, H. G. et al. Changes in Thickness and Surface Area of the Human Cortex and Their Relationship with Intelligence. Cerebral Cortex 25, 1608-1617 (2015).

27. Mills, K. L. et al. Inter-individual variability in structural brain development from late childhood to young adulthood. Neuroimage 242, 118450-118450 (2021).

28. Davidian, M. \& Giltinan, D. M. Nonlinear models for repeated measurement data: An overview and update. Journal of Agricultural, Biological, and Environmental Statistics 8, 387 (2003).

29. Reed, G. F., Lynn, F. \& Meade, B. D. Use of coefficient of variation in assessing variability of quantitative assays. Clin Diagn Lab Immunol 9, 1235-1239 (2002). 
30. MacPherson, S. E. et al. Processing speed and the relationship between Trail Making Test-B performance, cortical thinning and white matter microstructure in older adults. Cortex 95, 92-103 (2017).

31. Fuhrmann, D., Knoll, L. J. \& Blakemore, S. J. Adolescence as a sensitive period of brain development. Trends in cognitive sciences (2015) doi:10.1016/j.tics.2015.07.008.

32. Frankenhuis, W. E. \& Walasek, N. E. Evolution of sensitive periods: Bridging theory and data. Developmental Cogntitive Neuroscience (in press).

33. Baum, G. L. et al. Modular segregation of structural brain networks supports the development of executive function in youth. Current Biology (2017) doi:10.1016/j.cub.2017.04.051.

34. Baum, G. L. et al. Development of structure-function coupling in human brain networks during youth. Proc Natl Acad Sci USA 117, 771 (2020).

35. Power, J. D., Fair, D. A., Schlaggar, B. L. \& Petersen, S. E. The development of human functional brain networks. Neuron 67, 735-748 (2010).

36. Oldham, S. \& Fornito, A. The development of brain network hubs. Developmental Cognitive Neuroscience 36, 100607 (2019).

37. Ferschmann, L., Bos, M., Herting, M., Mills, K. L. \& Tamnes, C. K. Contextualizing adolescent structural brain development. (2021) doi:10.31234/osf.io/w8jq6.

38. Lenroot, R. K. et al. Sexual dimorphism of brain developmental trajectories during childhood and adolescence. Neurolmage 36, 1065-1073 (2007).

39. Wierenga, L. M. et al. A Key Characteristic of Sex Differences in the Developing Brain: Greater Variability in Brain Structure of Boys than Girls. Cerebral Cortex 28, 2741-2751 (2017). 
40. Goddings, A.-L., Beltz, A., Peper, J. S., Crone, E. A. \& Braams, B. R. Understanding the Role of Puberty in Structural and Functional Development of the Adolescent Brain. Journal of Research on Adolescence 29, 32-53 (2019).

41. Hyde, J. S. Gender Similarities and Differences. Annu. Rev. Psychol. 65, 373-398 (2014).

42. Casey, B. J. et al. The Adolescent Brain Cognitive Development (ABCD) study: Imaging acquisition across 21 sites. Developmental Cognitive Neuroscience 32, 43-54 (2018).

43. Tamnes, C. K. et al. Brain development and aging: Overlapping and unique patterns of change. Neurolmage 68, 63-74 (2013).

44. Madsen, K. S. et al. Maturational trajectories of white matter microstructure underlying the right presupplementary motor area reflect individual improvements in motor response cancellation in children and adolescents. Neurolmage 220, 117105 (2020).

45. Angstmann, S. et al. Microstructural asymmetry of the corticospinal tracts predicts right-left differences in circle drawing skill in right-handed adolescents. Brain Structure and Function 221, 4475-4489 (2016).

46. Gonzalez, M. R. et al. Brain structure associations with phonemic and semantic fluency in typically-developing children. Dev Cogn Neurosci 50, 100982-100982 (2021).

47. Madsen, K. S., Jernigan, T. L., Vestergaard, M., Mortensen, E. L. \& Baaré, W. F. C. Neuroticism is linked to microstructural left-right asymmetry of fronto-limbic fibre tracts in adolescents with opposite effects in boys and girls. Neuropsychologia 114, 1-10 (2018).

48. Madsen, K. S. et al. Brain microstructural correlates of visuospatial choice reaction time in children. Neurolmage 58, 1090-1100 (2011). 
49. Klarborg, B. et al. Sustained attention is associated with right superior longitudinal fasciculus and superior parietal white matter microstructure in children. Hum Brain Mapp 34, 3216-3232 (2013).

50. Plachti, A. et al. Only females show a stable association between neuroticism and microstructural asymmetry of the cingulum across childhood and adolescence: $\mathrm{A}$ longitudinal DTI study. bioRxiv 2021.08.31.458188 (2021) doi:10.1101/2021.08.31.458188.

51. Dale, A. M. \& Sereno, M. I. Improved Localizadon of Cortical Activity by Combining EEG and MEG with MRI Cortical Surface Reconstruction: A Linear Approach. Journal of Cognitive Neuroscience 5, 162-176 (1993).

52. Dale, A. M., Fischl, B. \& Sereno, M. I. Cortical Surface-Based Analysis: I. Segmentation and Surface Reconstruction. Neurolmage 9, 179-194 (1999).

53. Desikan, R. S. et al. An automated labeling system for subdividing the human cerebral cortex on MRI scans into gyral based regions of interest. Neurolmage 31, 968-980 (2006).

54. Reuter, M., Schmansky, N. J., Rosas, H. D. \& Fischl, B. Within-subject template estimation for unbiased longitudinal image analysis. Neuroimage 61, 1402-1418 (2012).

55. Rosen, A. F. G. et al. Quantitative assessment of structural image quality. Neuroimage 169, 407-418 (2018).

56. Comets, E., Lavenu, A. \& Lavielle, M. Parameter Estimation in Nonlinear Mixed Effect Models Using saemix, an R Implementation of the SAEM Algorithm. Journal of Statistical Software; Vol 1, Issue 3 (2017) (2017) doi:10.18637/jss.vo8o.io3. 
57. An, H., Landis, J. T., Bailey, A. G., Marron, J. S. \& Dittmer, D. P. drupl: A Stable Convergence Algorithm for the 4 Parameter Logistic Model. The R Journal 11, 171-190 (2019).

58. Rosseel, Y. lavaan: An R package for Structural Equation Modeling. Journal of Statistical Software 48, 1-36 (2012).

59. The Personality Project's Guide to R. http://personality-project.org/r/psych/.

6o. Shaw, P. et al. Neurodevelopmental trajectories of the human cerebral cortex. J. Neurosci. 28, 3586-3594 (2008).

61. Watson, C. G. brainGraph: Graph Theory Analysis of Brain MRI Data. (2020). 